\title{
Servo-Controlled Pneumatic Pressure Oscillator for Respiratory Impedance Measurements and High-Frequency Ventilation
}

\author{
DAVID W. KACZKA ${ }^{1}$ and KENNETH R. LUTCHEN ${ }^{2}$ \\ ${ }^{1}$ Department of Anesthesiology and Critical Care Medicine, Johns Hopkins University School of Medicine, Baltimore, MD; and \\ ${ }^{2}$ Department of Biomedical Engineering, Boston University College of Engineering, Boston, MA
}

(Received 3 November 2002; accepted 17 November 2003)

\begin{abstract}
The ability to provide forced oscillatory excitation of the respiratory system can be useful in mechanical impedance measurements as well as high frequency ventilation (HFV). Experimental systems currently used for generating forced oscillations are limited in their ability to provide high amplitude flows or maintain the respiratory system at a constant mean pressure during excitation. This paper presents the design and implementation of a pneumatic pressure oscillator based on a proportional solenoid valve. The device is capable of providing forced oscillatory excitations to the respiratory system over a bandwidth suitable for mechanical impedance measurements and HVF. It delivers high amplitude flows ( $>1.4 \mathrm{l} / \mathrm{s}$ ) and utilizes a servo-control mechanism to maintain a load at a fixed mean pressure during simultaneous oscillation. Under open-loop conditions, the device exhibited a static hysteresis of approximately $7 \%$, while its dynamic magnitude and phase responses were flat out to $10 \mathrm{~Hz}$. Broad-band measurement of total harmonic distortion was approximately $19 \%$. Under closed-loop conditions, the oscillator was able to maintain a mechanical test load at both positive and negative mean pressures during oscillatory excitations from 0.1 to $10.0 \mathrm{~Hz}$. Impedance of the test load agreed closely with theoretical predictions. We conclude that this servo-controlled oscillator can be a useful tool for respiratory impedance measurements as well as HFV.
\end{abstract}

Keywords-Proportional solenoid valve, Closed-loop pressure control, Forced oscillations, Harmonic distortion.

\section{INTRODUCTION}

The measurement of respiratory input impedance, the complex ratio of transrespiratory (or transpulmonary) pressure to flow at the airway opening as a function of frequency, is becoming an increasingly popular method for assessing the dynamic mechanical status of the lungs. When measured over low frequencies $(0.1-10 \mathrm{~Hz})$, respiratory impedance can be a sensitive indicator of serial and parallel airway heterogeneity, ${ }^{19}$ provides insight into the locus of airway constriction, ${ }^{18}$ and may be useful in partitioning the mechanical properties of airways and lung tissues. ${ }^{15}$

Address correspondence to David W. Kaczka, MD, PhD, Department of Anesthesiology and Critical Care Medicine, Johns Hopkins University School of Medicine, Blalock 1412, 600 North Wolfe Street, Baltimore, MD 21287. Electronic mail: dkaczka1@jhmi.edu
Several approaches have been developed to measure low frequency respiratory impedance in humans and large animals. The most common is to excite the respiratory system with small amplitude pseudorandom noise using a loud-speaker. ${ }^{9}$ While straightforward, this technique has several technical and clinical drawbacks. It requires highperformance subwoofer speakers relatively free of harmonic distortion. Moreover, only nonphysiologic flows can be generated (typically less than $0.2 \mathrm{l} / \mathrm{s}$ ) that are often load-dependent unless a closed-loop design is employed. ${ }^{3,5}$ Finally, this approach requires considerable subject cooperation. Awake subjects require training to achieve the necessary prolonged periods of apnea and respiratory muscle relaxation, ${ }^{9}$ which makes the method impractical for routine use in patients with impaired lung function. In anesthetized and paralyzed patients, this technique usually requires temporary interruption of artificial ventilatory support. ${ }^{23}$

More recent studies have incorporated high amplitude broad-band flow forcings into waveforms that mimic physiological breathing maneuvers. ${ }^{12,13,15,20}$ Specifically, Optimal Ventilator Waveforms $(\mathrm{OVWs})^{20}$ and Enhanced Ventilator Waveforms $(\mathrm{EVWs})^{14}$ concentrate flow spectral energy at specific frequencies to minimize nonlinear harmonic distortion in the resulting pressure waveforms. The phases of these waveforms are optimized to achieve tidal volume excursions sufficient for gas exchange, and thus are more clinically appropriate for awake and anesthetized patients. ${ }^{12,13,15}$ Presently, these waveforms must be generated by piston-cylinder arrangements actuated by servocontrolled linear motors, allowing for delivery of high amplitude and load-independent oscillatory flows. ${ }^{15,20,27,28}$ Despite the ability of such systems to produce high fidelity flow waveforms, ${ }^{15,27}$ they can be extremely inefficient due to mechanical friction and stick-slip effects between the piston and cylinder. ${ }^{1}$ Moreover, their use in humans or large animals require high-powered electrical driving amplifiers, capable of dissipating over $200 \mathrm{~W}^{28}$

Regardless of the method employed to acquire respiratory impedance data, a more vexing problem is the ability of either approach to make oscillatory measurements 
while the lungs are maintained at a specified mean volume or pressure. Since lung volume can significantly impact respiratory impedance, ${ }^{22}$ the ability to provide forced oscillations at different lung volumes or mean airway pressures is useful in understanding the impact of PEEP, periodic sighs, and recruitment/derecruitment manuevers on dynamic lung mechanics. Previous forced oscillation studies have relied on the manual adjustment of small bias flows into the breathing circuit to compensate for leaks and to maintain specified lung volumes during oscillatory excitations. ${ }^{2,13,15}$ While several investigators have developed closed-loop systems to maintain mean airway pressure during forced oscillations, ${ }^{3,5,7,28,29}$ they must rely on separate servo-controlled systems operating in parallel to an oscillatory pressure or flow generator.

In addition to the diagnostic information that forced oscillations provide, such excitations can be therapeutic as well. High frequency ventilation (HFV) is becoming a standard of care in neonatal lung injury, and there has been renewed interest in using this ventilatory modality in the treatment of pediatric and adult lung injury as well. ${ }^{16} \mathrm{In}$ contrast to conventional mechanical ventilation, HFV maintains gas exchange using small tidal volumes (often less than anatomic dead space) delivered at supraphysiologic rates (i.e., 5-15 Hz). However, the use of HFV in clinical environments requires fine control over both the mean level of airway pressure as well as the amplitude of peak-to-peak pressure oscillations.

Given the limitations of devices currently available for generating forced oscillations in humans and large animals, our goal was to design and implement an oscillator capable of delivering physiological flows and tidal volumes over a frequency range sufficient for impedance measurements and HFV. The specific design goals of this prototype device were: 1) the capability for high amplitude flow delivery $(>1.4 \mathrm{l} / \mathrm{s})$ with a dynamic response suitable for low frequency respiratory impedance measurements as well as HFV; 2) utilization of a servo-control mechanism to maintain the respiratory system at a specified mean pressure during oscillatory excitation; and 3 ) the minimization of electrical power consumption compared to traditional linear motor driven devices used for the same purposes. In addition, we investigated the static and dynamic performance and linearity of the device over a bandwidth appropriate for respiratory impedance measurements as well as HFV. The ability of the device to maintain a mechanical test load at a specified mean pressure during oscillatory excitation was assessed under closed-loop conditions. Finally the device was used to measure the impedance of a test load, the results of which were compared to theoretical predictions.

\section{OVERVIEW OF PNEUMATIC PRESSURE OSCILLATOR}

The pneumatic pressure oscillator is schematically depicted in Fig. 1. The key component of this system is its pro- portional solenoid (PSOL) valve (ASCO Posiflow ${ }^{\mathrm{TM}}$ Model SD8202G4V, Florham Park, NJ). This valve has a maximum orifice diameter of $5 / 32^{\prime \prime}$, and is capable of delivering up to $\sim 3.0 \mathrm{l} / \mathrm{s}$ for a 10 PSI pressure drop. Flow through the valve is determined by the position of its spring-loaded core. An electric current applied to the solenoid coil generates an electromagnetic pullforce on the core. When this pullforce exceeds the opposing spring force, the core moves upward and opens the valve. The degree to which the valve opens is thus proportional to the current applied to the coil. Accurate positioning of the core is provided by ASCO's Electronic Control Unit (ECU) Model 8908001, which converts a $0-10 \mathrm{~V}$ control signal to a $24 \mathrm{~V}$ pulse-width modulated coil-excitation signal. The average current through the coil and the amplitude of the current variations is dependent on the switching frequency of the pulse-width modulator (factory configured to $300 \mathrm{~Hz}$ ). The ECU provides additional control of the current to compensate for any temperaturedependent changes in coil resistance. The combined ECUPSOL system is powered electrically by a single 24 VDC linear power supply (Power-One HC24-2.4-A, Camarillo, $\mathrm{CA})$, and consumes less than $23 \mathrm{~W}$ of electrical power.

A pressure regulator (Bellofram P/N 231-960-956-000, Newell, WV) steps down a standard 50 PSI wall source pressure to a 10 PSI driving pressure for the PSOL. To achieve bidirectional flows, a $21^{\prime \prime} \mathrm{Hg}$ suction line with an adjustable needle valve was added immediately after the solenoid valve output nozzle. The high input impedance of this line is was sufficient to minimize the shunting of oscillatory flows through it. By matching the steady flow though this sink $\left(\dot{V}_{\text {sink }}\right)$ to the mean flow directly out of the proportional solenoid valve $\left(\dot{V}_{\text {psol }}\right)$, the resulting flow delivered to the load impedance $\left(\dot{V}_{\text {load }}\right)$ becomes purely oscillatory or bidirectional. The entire device is housed in a $28 \times 30 \times 14 \mathrm{~cm}$ metal enclosure which is electrically grounded (Fig. 2).

A block diagram of the entire system is illustrated in Fig. 3. Control voltages corresponding to the desired mean pressure $\left(\nu_{\mathrm{P}}^{\text {mean }}\right)$ and desired oscillatory pressure $\left(\nu_{\mathrm{P}}^{\text {osc }}\right)$ are set by the operator. The actual load pressure $(P)$ is electrically transduced ( $\left.K_{\text {trans }}\right)$, low-pass filtered (LPF), and compared to the total desired pressure signal. Since the frequency response of the entire closed-loop system depends on the mechanical load impedance $\left(Z_{\text {load }}\right)$ under excitation, a proportional controller $\left(K_{\mathrm{P}}\right)$ was added to adjust the amplitude of the error signal before it was presented to the ECU. Alternatively, direct open-loop excitation of the ECU-PSOL system is possible with the servo enable switch opened. Here, the user may apply control voltages corresponding to an offset adjustment $\left(\nu_{\dot{v}}^{\text {offset }}\right)$ or an oscillatory flow component $\left(\nu_{\dot{v}}^{\text {osc }}\right)$. Such an open-loop arrangement may be useful when precise control of mean load pressure is not needed, as when measuring the impedance of a cylinder or pipe opened to the atmosphere. An open-loop configuration may also be desirable if an expiratory valve system is incorporated into the device to allow a patient-driven exhalation to the 


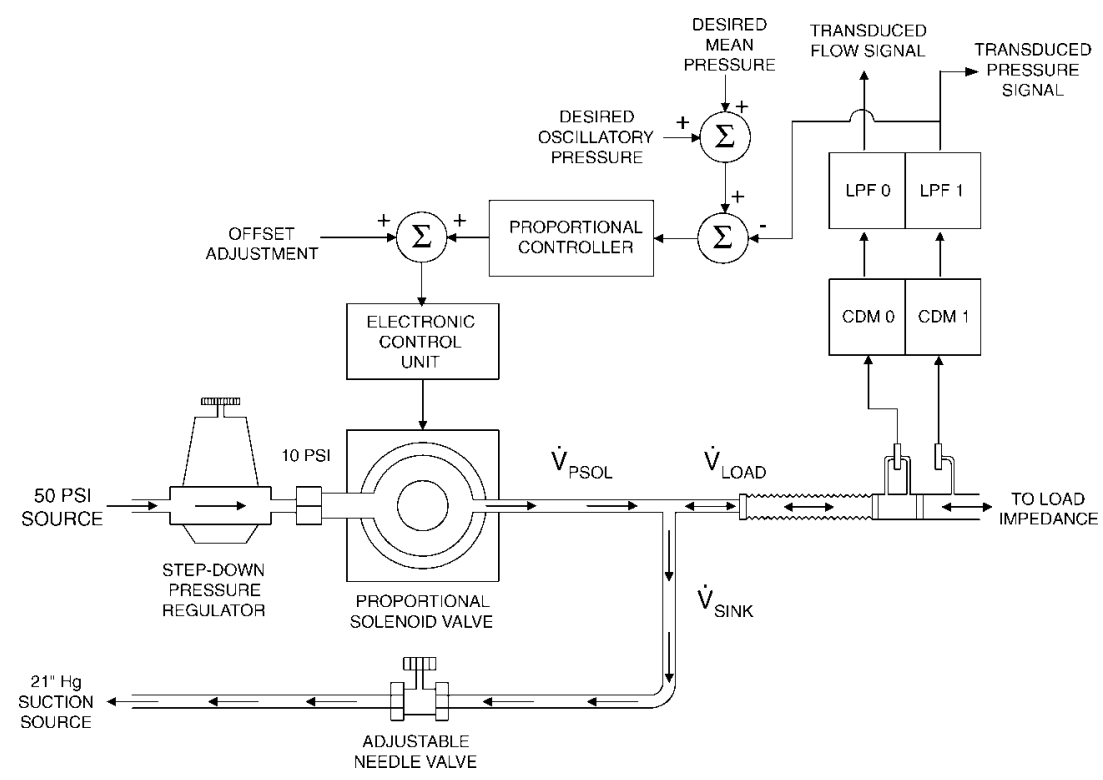

FIGURE 1. Schematic of pneumatic pressure oscillator. LPF: low pass filter; CDM: carrier demodulator; $\dot{V}_{\text {psol }}:$ total flow from proportional solenoid valve; $\dot{V}_{\text {sink }}$ : suction flow; $\dot{V}_{\text {load }}$ : net flow delivered to load. See text for details.

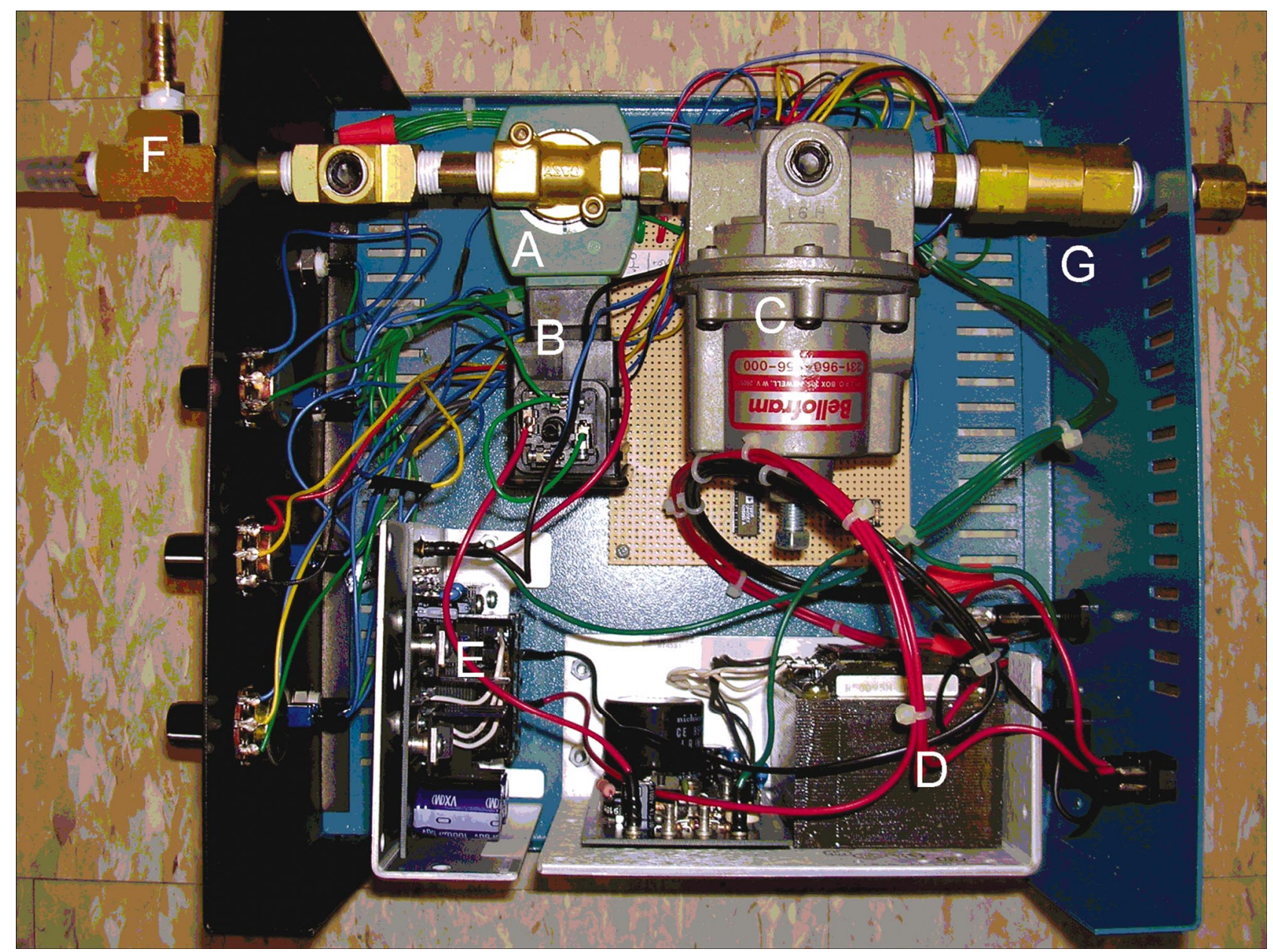

FIGURE 2. Overhead view of complete pneumatic pressure oscillator housed in its metal enclosure. (A) proportional solenoid valve; (B) electronic control unit; (C) pressure regulator; (D) $24 \mathrm{~V}$ linear power supply; (E) $\pm 15 \mathrm{~V}$ linear power supply; (F) system output; (G) check valve. 


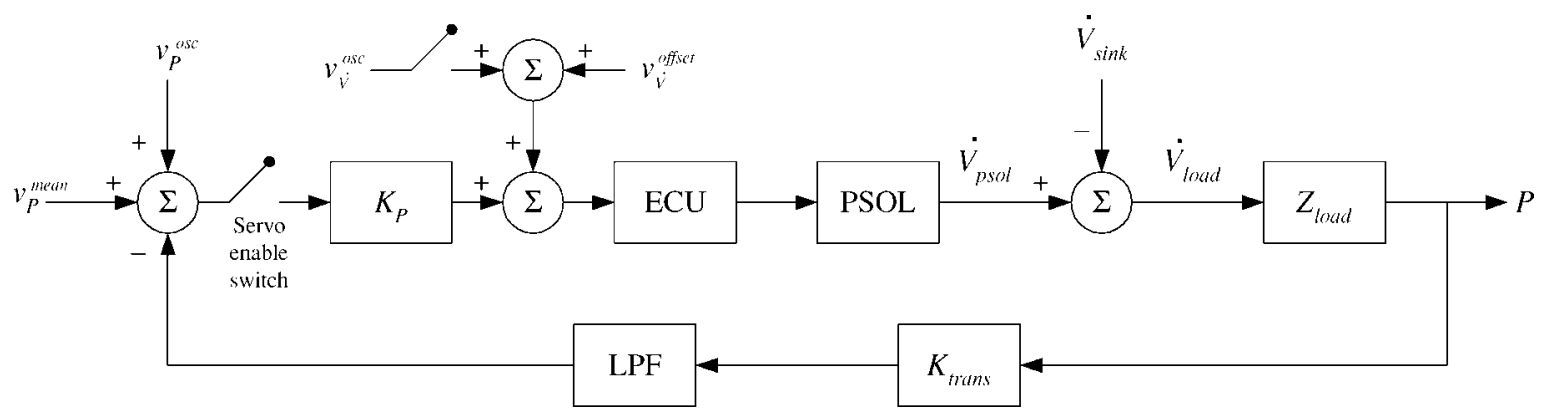

FIGURE 3. Block diagram of pneumatic pressure oscillator. $v_{P}^{\text {mean }}$ : control voltage for desired mean pressure; $\nu_{\mathrm{P}}^{\mathrm{osc}}$ : control voltage for desired oscillatory pressure; $\nu_{\dot{V}}^{\text {offset }}$ : control voltage for offset flow adjustment; $\nu_{\dot{v}}^{\text {osc }}$ : control voltage for oscillatory flow; $K_{\mathrm{P}}$ : proportional controller gain; ECU: electronic control unit; PSOL: proportional solenoid valve; $\dot{V}_{\text {psol: }}$ total flow from proportional solenoid valve; $\dot{V}_{\text {sink }}$ : suction flow; $\dot{V}_{\text {load }}$ : net flow delivered to load; $Z_{\text {load }}$ : load impedance; $P$ : load pressure; $K_{\text {trans }}$ : pressure transducer gain; LPF: low pass filter.

atmosphere or against PEEP. ${ }^{14}$ In this situation, direct excitation of the PSOL occurs during inspiration. Mean airway pressure will not be servo-controlled and will instead be a function of both peak and end-expiratory pressures as well as breathing frequency.

Figure 4 illustrates the electronics schematic for the servo-control circuit. OP-400 op-amps are used in the circuit, which is entirely powered by a single $12 \mathrm{~W} \pm 15 \mathrm{VDC}$ linear power supply (Power-One HAD15-0.4-A, Camarillo, $\mathrm{CA})$. The user adjusts the voltage corresponding to the desired mean pressure level with potentiometer RP-1. This signal is then buffered by op-amp OA-1 and summed by OA-2 to the voltage corresponding to the desired oscillatory pressure component. The total desired pressure signal is then inverted by OA-3, the output of which is connected in parallel to an external female BNC adapter for viewing on an oscilloscope. This signal is then compared at OA-5 to the actual transduced pressure signal buffered by OA4. Potentiometer RP-3 adjusts the gain of the proportional controller. The amplified error signal is then added at OA-7 to a $0-10 \mathrm{~V}$ offset DC-voltage ( $\left.\nu_{\mathrm{V}}^{\text {offset }}\right)$ which is adjusted at
RP-2 to ensure that the PSOL is operating about is linear region. An additional summing junction is available (SW-2) for direct external excitation of the ECU-PSOL system. A servo enable switch (SW-3) may be thrown when closedloop control of the load pressure is desired. The entire actuating signal is then inverted at OA- 8 before being passed on to the ECU.

\section{PERFORMANCE EVALUATION}

\section{Steady-State Performance of Oscillator}

The steady-state linearity of the PSOL valve was assessed first by presenting a DC voltage to the ECU and measuring the corresponding flow output from the solenoid using a calibrated pneumotachograph (Hans Rudolph 4700A, Kansas City, MO) connected to a $0-2 \mathrm{~cm} \mathrm{H}_{2} \mathrm{O}$ variable reluctance pressure transducer (Celesco LCVR-0002, Canoga Park, CA). Input voltage was first increased in $0.1-0.4 \mathrm{~V}$ increments up to $12 \mathrm{~V}$, then decreased back down to $0 \mathrm{~V}$ in a similar fashion. Control voltages to the ECU were

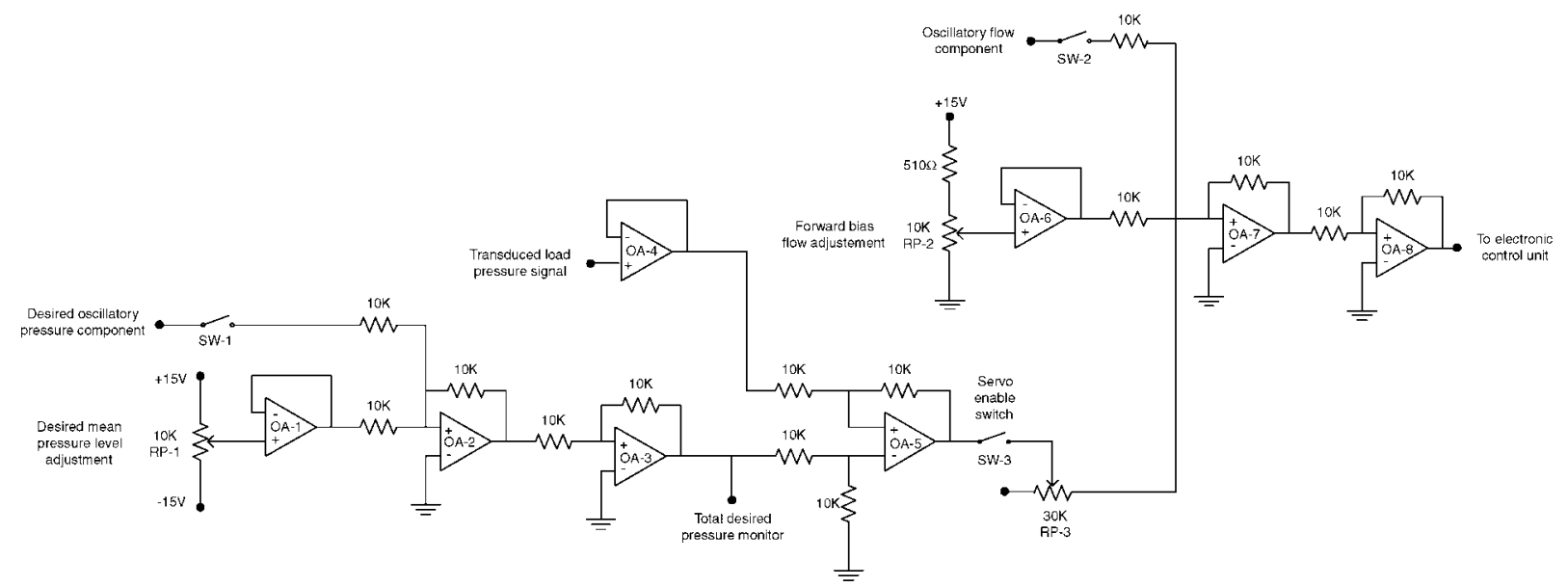

FIGURE 4. Electronics schematic diagram for the servo-control circuit. All operational amplifiers are OP-400 s. The entire circuit is powered by $a \pm 15 \mathrm{~V}$ linear power supply. See text for details. 
measured by a digital multimeter (Tektronix DM511, Heerveen, The Netherlands). Hysteresis of the combined ECU-PSOL system was determined as the maximum difference in flow over this single calibration cycle expressed as a percent of the full-scale flow.

\section{Dynamic Performance of Oscillator-Open Loop}

To evaluate the load-free performance of the PSOL valve in terms of the quality of its generated flow waveforms, we determined its open-loop dynamic response using a pseudorandom signal consisting of 15 sinusoids with equivalent amplitudes and random phases. The frequency components were chosen to obey the non-sum non-difference (NSND) criteria of Suki and Lutchen (30) such that the impact of harmonic distortion and cross-talk in the system output would be minimized. A 2048 point NSND waveform with energy concentrated between 0.098 and $40.97 \mathrm{~Hz}$ was generated with a digital-to-analog converter (Data Translations DT2811, Marlboro, MA) at a shift frequency of $100 \mathrm{~Hz}$. The output of the D/A converter was low pass filtered at $50 \mathrm{~Hz}(8$ pole Butterworth, Ithaco) and electronically summed with a 5-V DC component (at OA-6 in Fig. 4) to ensure operation of the PSOL about its mid-range to minimize the effects of saturation nonlinearities (see Fig. 5 in Results section). The output nozzle of the PSOL was opened to atmosphere, and flow was measured using the same pneumotach arrangement described above. Both the input voltage and output flow signals were low pass filtered at $50 \mathrm{~Hz}$, sampled at $100 \mathrm{~Hz}$ with an analog-to-digital converter (Data Translations DT-2811). The amplitude of the D/A output was adjusted to achieve peak NSND voltages of $0.4,0.8,1.2,1.6$, and $2.0 \mathrm{~V}$ which were presented to the ECU in random order. The open-loop transfer function of the combined ECUPSOL system was determined from the ratio of the cross power spectrum of the input voltage and output flow to the autopower spectrum of voltage. After neglecting the first three transient NSND cycles, six 20.48-s rectangular windows with $83 \%$ overlap were used to calculate the transfer function, which was expressed in polar coordinates. Measurements were made with and without the suction in-line to determine the impact of suction on the dynamics and linearity on the output flow.

To quantify dynamic nonlinear harmonic distortion and cross-talk of the open-loop system, we used an harmonic distortion index $\left(k_{\mathrm{d}}\right)$ appropriate for broad-band excitations: ${ }^{32}$

$$
k_{\mathrm{d}}=100 \% \times \sqrt{\frac{P_{\mathrm{NI}}}{P_{\mathrm{TOT}}}}
$$

where $P_{\text {TOT }}$ is the total power in the signal (i.e., sum of squared magnitudes in the frequency-domain) and $P_{\mathrm{NI}}$ is the power at noninput (i.e., non-NSND) frequencies. To determine the impact of nonlinearities and noise present at non-NSND frequencies in the analogue input driving signal, we calculated $k_{\mathrm{d}}$ for both the input voltage and output flow waveforms.

\section{Dynamic Performance of Oscillator-Closed Loop}

The closed-loop performance of the system was assessed using a simulated mechanical load impedance consisting of a screen-mesh resistor in series with a 201 glass bottle packed with copper wool to minimize the temperature changes associated with cyclic gas compression. The resistive load ( $R_{\text {load }}$ ) of the screen-mesh was experimentally determined to be approximately $4 \mathrm{~cm} \mathrm{H}_{2} \mathrm{O} / \mathrm{l} / \mathrm{s}$. The elastic load $\left(E_{\text {load }}\right)$ provided by the bottle was determined from Boyle's Law:

$$
E_{\mathrm{load}}=\beta \frac{P_{0}}{V_{0}}
$$

where $P_{0}$ represents the absolute mean bottle pressure (approximately $1033 \mathrm{~cm} \mathrm{H}_{2} \mathrm{O}$ for $\left.1 \mathrm{~atm}\right), V_{0}$ represents the compressible volume of the chamber (201 in our case), and $\beta$ is a constant equal to 1.0 for isothermal compression and 1.4 for adiabatic compression. ${ }^{25,27}$ Assuming a mean bottle pressure of zero relative to atmosphere, we computed the theoretical elastic loads of the bottle as 51.68 and $72.35 \mathrm{~cm}$ $\mathrm{H}_{2} \mathrm{O} / 1$ for isothermal and adiabatic compression, respectively. The input driving signal to the system was identical to the NSND waveform described above.

Peak oscillatory NSND control voltage amplitudes were adjusted to 1, 2, 3, 4, and $5 \mathrm{~V}$ and applied in random order. The mean load pressure was maintained at $0 \mathrm{~cm} \mathrm{H}_{2} \mathrm{O}$. A 0-50 $\mathrm{cm} \mathrm{H} \mathrm{H}_{2} \mathrm{O}$ pressure transducer (Celesco LCVR-0050) was located proximal to the screen resistor for measurement and feedback of the load pressure. The proportional controller gain $K_{\mathrm{P}}$ was set to approximately 0.3 by adjusting the RP-3 potentiometer of Fig. 4. Both the desired and actual pressure signals were sampled and processed as described previously. The closed-loop transfer function of the system was computed using the cross-power spectral method with the actual load pressure as the system output. The $k_{\mathrm{d}}$ indices were also computed for the input control voltage and output load pressure.

\section{Dynamic Performance of Oscillator-Mean Pressure Control}

The ability of the closed-loop system to maintain a load at a given desired mean pressure during simultaneous oscillatory excitations was assessed by driving the system with $0.1,1.0$, and $10.0 \mathrm{~Hz}$ sine waves while maintaining the mean pressure level of the resistor-bottle load at $-10,0$, and $10 \mathrm{~cm}$ $\mathrm{H}_{2} \mathrm{O}$. The different mean pressure levels were achieved by manually adjusting the RP-1 potentiometer of Fig. 4. Sine waves were generated by the D/A board at shift frequency of $100 \mathrm{~Hz}$ and low-pass filtered at $50 \mathrm{~Hz}$. The corresponding load pressure was also low-pass filtered at $50 \mathrm{~Hz}$ and sampled at $100 \mathrm{~Hz}$ by the A/D board. To test the ability 
of the system to follow dynamic changes in desired mean pressure, we linearly increased the desired mean bottle pressure from $-15 \mathrm{~cm} \mathrm{H}_{2} \mathrm{O}$ to $+15 \mathrm{~cm} \mathrm{H}_{2} \mathrm{O}$ over a period of $27 \mathrm{~s}$ while simultaneously oscillating the load at frequencies of 1.0 and $10.0 \mathrm{~Hz}$.

\section{Impedance Measurements}

Mechanical impedance of the test load was measured using the same 2048 point NSND waveform as the input driving pressure signal to the system with mean load pressures adjusted to $-10,0$, and $+10 \mathrm{~cm} \mathrm{H}_{2} \mathrm{O}$. The delivered flow and load pressure were transduced as detailed earlier, although both signals were low pass filtered at $10 \mathrm{~Hz}$ prior to sampling. $Z_{\text {load }}$ was determined from the ratio of the cross-power spectrum of the load pressure and flow to the autopower spectrum of flow, again using a 20.48 s rectangular window with $83 \%$ overlap. Load resistance $\left(R_{\text {load }}\right)$ and elastance $\left(E_{\text {load }}\right)$ at each specified NSND frequency $\left(f_{\mathrm{k}}\right)$ were computed from the real $(\mathrm{Re})$ and imaginary $(\mathrm{Im})$ components of $Z_{\text {load }}$ :

$$
\begin{gathered}
R_{\text {load }}\left(f_{\mathrm{k}}\right)=\operatorname{Re}\left\{Z_{\text {load }}\left(f_{\mathrm{k}}\right)\right\} \\
E_{\text {load }}\left(f_{\mathrm{k}}\right)=-2 \pi f_{\mathrm{k}} \operatorname{Im}\left\{Z_{\text {load }}\left(f_{\mathrm{k}}\right)\right\}
\end{gathered}
$$

\section{RESULTS}

\section{Steady-State Performance of Proportional Solenoid}

The steady-state voltage-flow curves for both increasing and decreasing control voltages are shown in Fig. 5. Both limbs were sigmoidal in nature, but were fairly linear over the 3-8 V range, corresponding to minimum and maxi- mum flows of approximately 0.2 to $2.5 \mathrm{l} / \mathrm{s}$, respectively. The PSOL valve exhibited approximately $7 \%$ hysteresis over the full range of flows from 0 to $2.85 \mathrm{l} / \mathrm{s}$.

\section{Dynamic Performance of PSOL_Open Loop}

Figure 6 shows the magnitude and phase response of the open-loop ECU-PSOL system for NSND peak amplitudes of $0.4,0.8,1.2,1.6$, and $2.0 \mathrm{~V}$, corresponding to peak-topeak output flows of $0.76,1.68,2.50,2.83$, and $2.991 / \mathrm{s}$, respectively. Measurements were made with and without suction. In both cases, the magnitude and phase responses are relatively flat out to $10 \mathrm{~Hz}$. For all amplitudes, the system consistently demonstrated a resonance at about $21 \mathrm{~Hz}$. While the magnitude response did demonstrate some variability below $10 \mathrm{~Hz}$ with amplitude, there was no consistent trend with increasing amplitude. The phase response demonstrated minimal variability regardless of NSND amplitude. Suction reduced much of the variability observed in the magnitude below $10 \mathrm{~Hz}$, but appeared to have little impact on the open-loop phase response of the PSOL.

Figure 7 shows the input voltage and output flow harmonic distortion indices as a function of input RMS voltage with and without suction. In both cases, $k_{\mathrm{d}}$ for the input control voltage was minimal, demonstrating an RMSdependent decrease below $0.2 \mathrm{~V} \mathrm{RMS}$, above which it became fairly constant and less than $2 \%$. The $k_{\mathrm{d}}$ for flow was substantially higher. With no suction applied, it averaged $28.05 \%$ with a standard deviation of $1.81 \%$. When suction was applied however, the flow $k_{\mathrm{d}}$ dropped significantly, averaging $19.90 \%$ with a standard deviation of $3.03 \%$, and

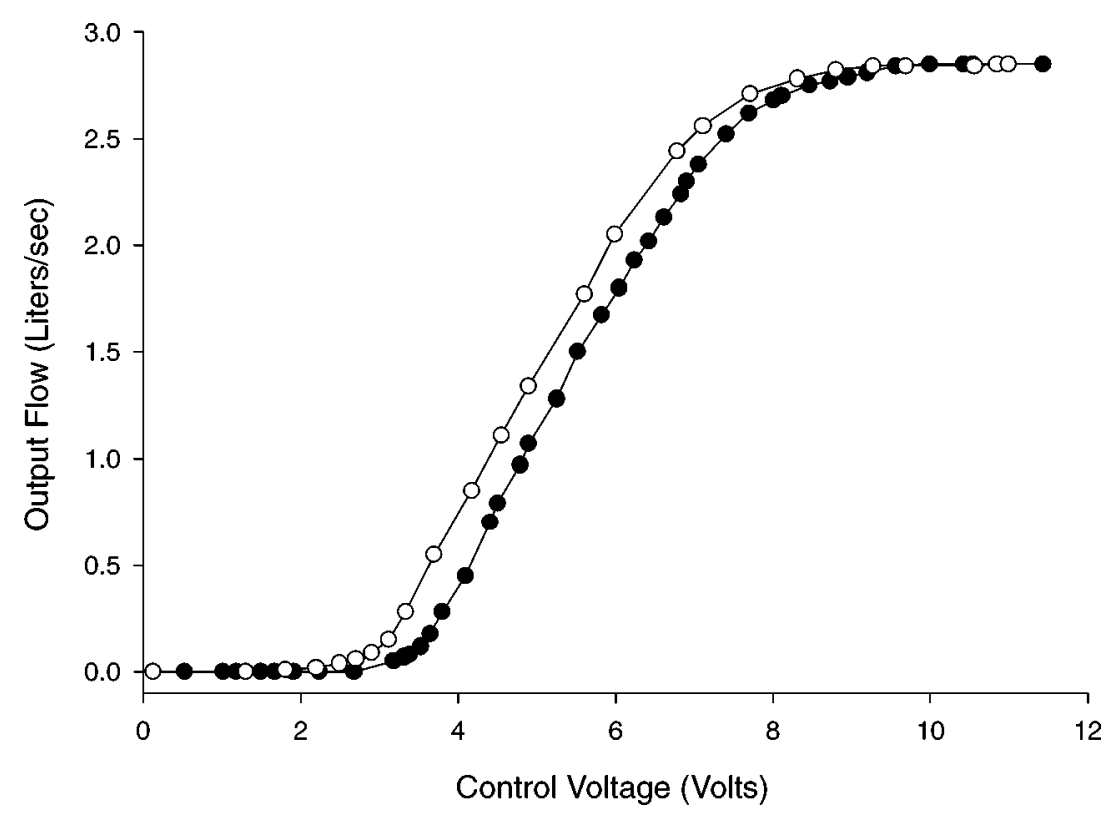

FIGURE 5. Steady-state voltage-flow curves of combined ECU-PSOL system for increasing (solid circles) and decreasing (open circles) control voltages. Hysteresis curve represents the result of a single cycle. 

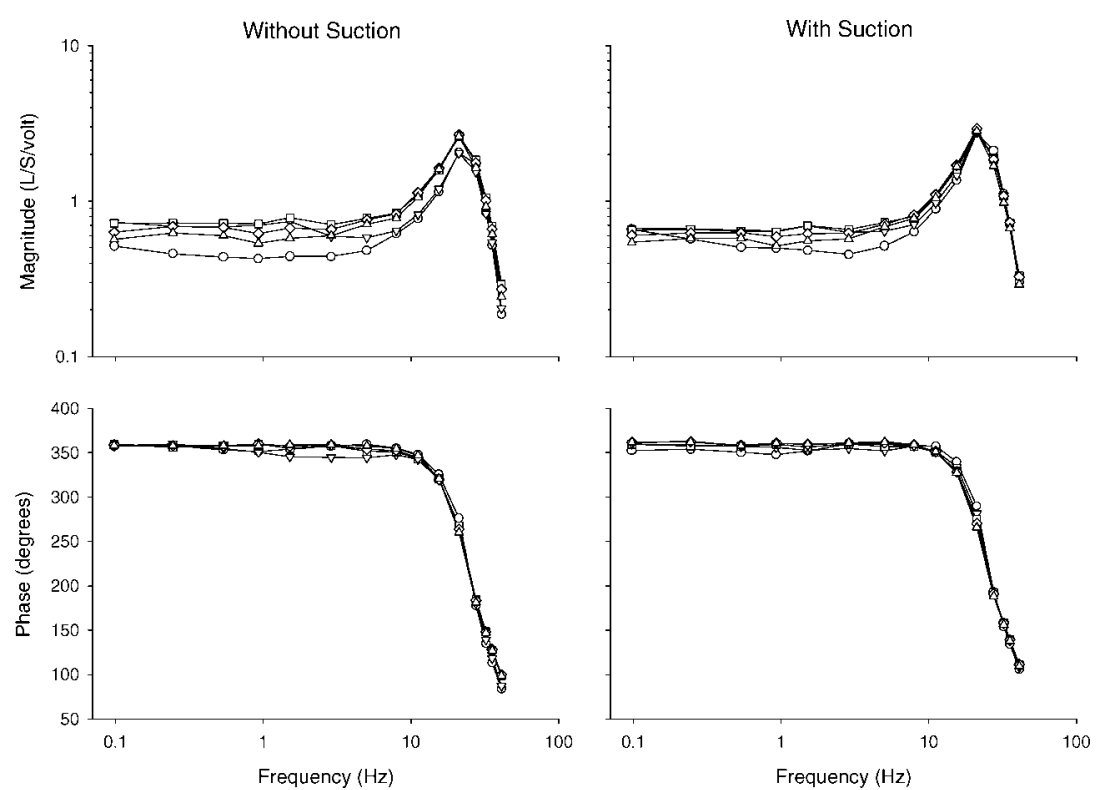

FIGURE 6. Magnitude and phase responses for combined ECU-PSOL open-loop systems with and without suction at peak D/A control voltages of 0.4 (circles), 0.8 (inverted triangles), 1.2 (squares), 1.6 (diamonds), and 2.0 (triangles) V.

exhibited a slight trend of increasing harmonic distortion with increasing RMS voltage.

\section{Dynamic Performance of Pressure Oscillator-Closed Loop}

Figure 8 shows the magnitude and phase plots for the closed-loop pressure oscillator from 0.098 to $40.97 \mathrm{~Hz}$ with peak NSND voltages of $1,2,3,4$, and $5 \mathrm{~V}$, corresponding to peak-to-peak pressures of 4.0, 9.9, 16.2, 21.2, and $24.9 \mathrm{~cm}$ $\mathrm{H}_{2} \mathrm{O}$. Measurements were made with the mechanical load at a mean pressure of $0 \mathrm{~cm} \mathrm{H}_{2} \mathrm{O}$ relative to atmosphere. In all cases, the magnitude reaches a minimum at about $5 \mathrm{~Hz}$, and thereafter increases to a maximum at about $21 \mathrm{~Hz}$. The phase response demonstrated a slight negative frequency dependence out to $1 \mathrm{~Hz}$. Beyond $1 \mathrm{~Hz}$, the phase gradually increased until about $11 \mathrm{~Hz}$, thereafter demonstrating a sharp frequency-dependent drop. There was a slight positive dependence of the closed-loop magnitude with increasing NSND amplitude, although this was not seen in the phase response.

Figure 9 shows the input voltage and output load pressure harmonic distortion indices. As seen previously for the open-loop experiments, the $k_{\mathrm{d}}$ for the input driving voltage was minimal, and again exhibited a negative dependence on RMS voltage. The $k_{\mathrm{d}}$ for the load pressure, however, ranged from 12.56 to $23.54 \%$, with no clear dependence on RMS voltage.
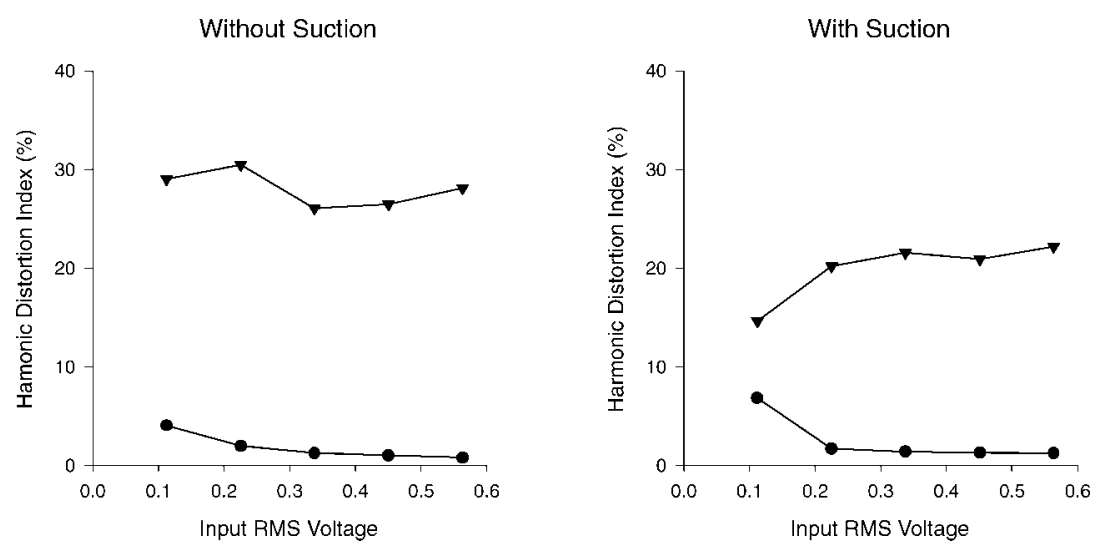

FIGURE 7. Input control voltage (circles) and output flow (inverted triangles) harmonic distortion indices for the combined ECUPSOL system as a function of input RMS voltage. 

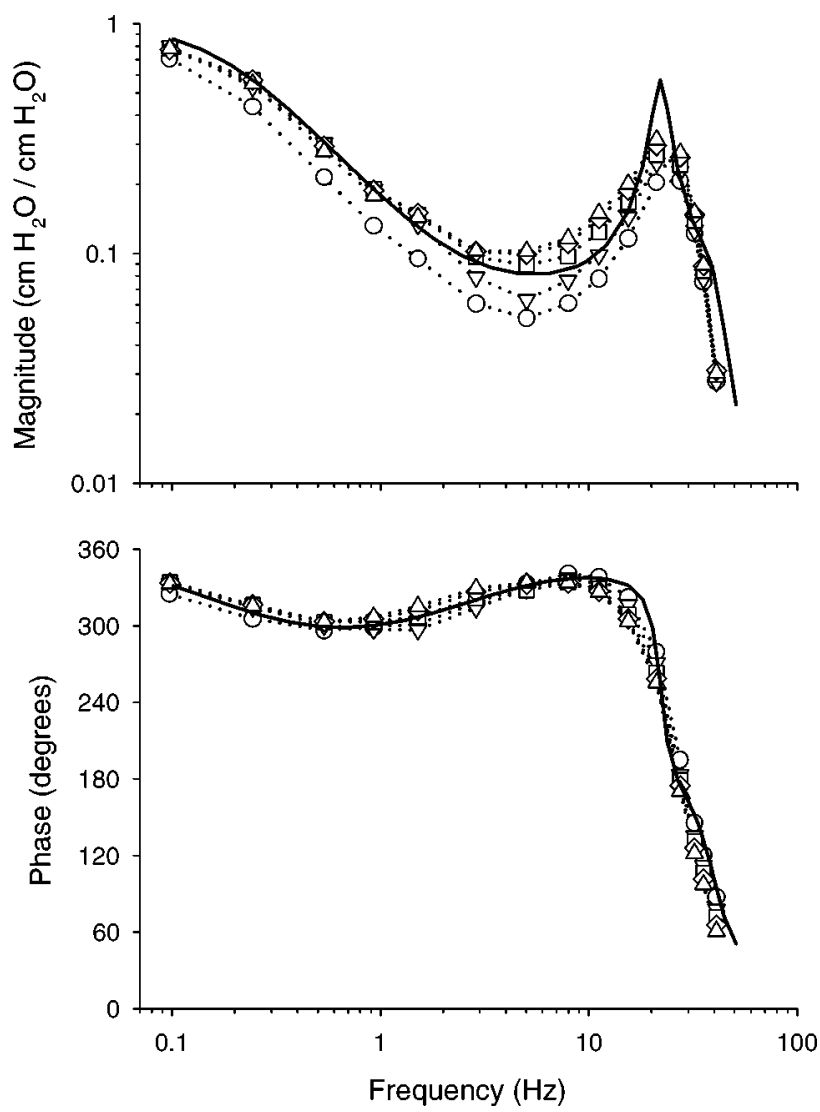

FIGURE 8. Magnitude and phase responses for closed-loop pressure oscillator at peak D/A control voltages of 1 (circles), 2 (inverted triangles), 3 (squares), 4 (diamonds), and 5 (triangles) volts with a $Z_{\text {load }}$ consisting of a screen resistance and $20 \mathrm{I}$ glass bottle. Solid line is a prediction of the frequency response for the closed-loop system (Fig. 3) using a computer simulation in which a mathematical model of the resistor and bottle load was connected to the output of transfer function describing the ECU-PSOL dynamics (Fig. 6). See Appendix for details.

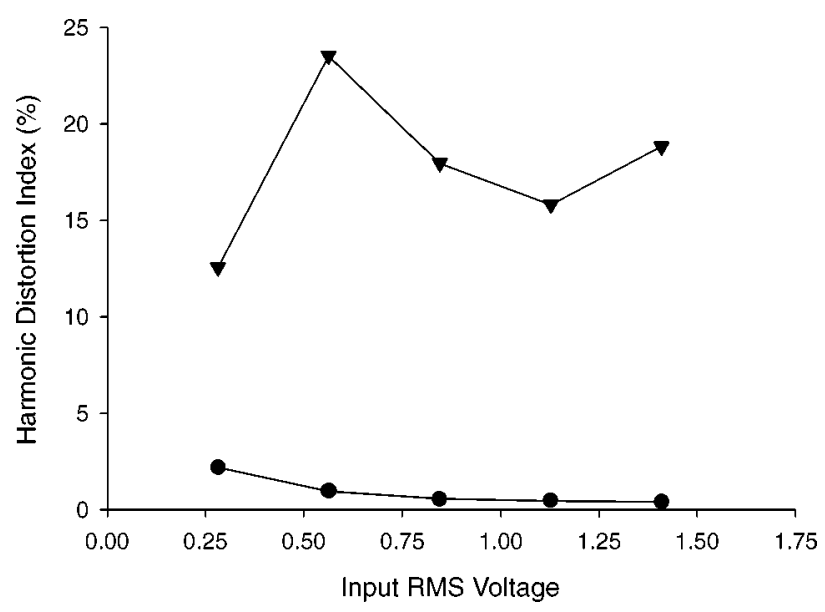

FIGURE 9. Input control voltage (circles) and output pressure (inverted triangles) harmonic distortion indices for the pneumatic pressure oscillator as a function of input RMS voltage.
Examples of the actual pressure tracings for the mechanical test load at three frequencies $(0.1,1.0$, and $10.0 \mathrm{~Hz})$ and three mean pressures $\left(-10,0\right.$, and $\left.+10 \mathrm{~cm} \mathrm{H}_{2} \mathrm{O}\right)$ are shown in Fig. 10. Consistent with the closed-loop bode plot of Fig. 8, the actual peak-to-peak amplitude of the pressure oscillations decreased with increasing frequency. However, the servo system was able to maintain the mean pressure at a constant level in all cases. The ability of the closedloop system to follow dynamic changes in the desired mean load pressure with superimposed 1 and $10 \mathrm{~Hz}$ oscillations is shown in Fig. 11.

\section{Impedance Measurements}

Figure 12 shows the measured resistive and elastic components of the mechanical test load from approximately $0.09-8 \mathrm{~Hz}$ at mean pressures of $-10,0$, and $+10 \mathrm{~cm} \mathrm{H}_{2} \mathrm{O}$. Also shown are the theoretical upper and lower limits for elastance, assuming isothermal gas compression at $-10 \mathrm{~cm}$ $\mathrm{H}_{2} \mathrm{O}$ and adiabatic gas compression at $+10 \mathrm{~cm} \mathrm{H}_{2} \mathrm{O}$, respectively. At all three mean load pressures, $R_{\text {load }}$ shows a frequency-dependent decrease which asymptotically approaches a value approximately equal to the screen resistance of $4 \mathrm{~cm} \mathrm{H}_{2} \mathrm{O} / \mathrm{l} / \mathrm{s}$. Except at the very highest frequency, $E_{\text {load }}$ was within its theoretical upper and lower limits, with all three curves demonstrating a trend of a increasing from isothermal to adiabatic compression as frequency increased.

\section{DISCUSSION}

The forced oscillation technique has evolved into powerful tool for the assessment of respiratory mechanics. It has the potential for routine use in pulmonary medicine for both diagnostic and therapeutic purposes. Respiratory impedance can provide much information about the mechanical status of the lungs, and HFV is becoming a popular ventilatory modality, especially in patients with the acute respiratory distress syndrome (ARDS).$^{16}$ Since ARDS is a mechanically heterogenous disease, it can be a challenge to maintain the delicate balance between oxygenation and overdistention injuries. While conventional mechanical ventilation may predispose certain regions of the lung to further injury with high tidal volumes and suboptimal endexpiratory pressures, HFV can maintain gas exchange at specified mean alveolar volumes while minimizing the impact of cyclic overdistention and shear stresses associated with intratidal derecruitment of lung volume. Nonetheless, there has yet to be a randomized clinical trial demonstrating patients receiving HFV have significantly better outcomes than those receiving conventional ventilation. Since this may be due to an incomplete understanding of the affects of mean airway pressure, oscillation amplitude, and frequency content on gas exchange in the injured lung, our device may be ideal for addressing such questions in clinical 

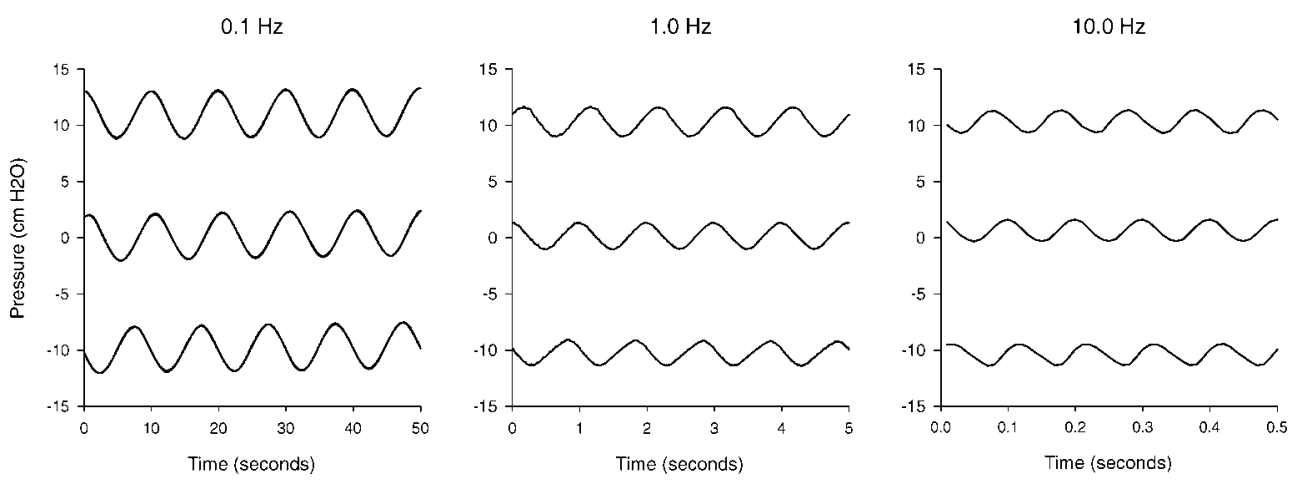

FIGURE 10. Actual pressure tracings for the resistor and glass bottle mechanical load at frequencies of $0.1,1.0$, and $10.0 \mathrm{~Hz}$ and mean pressures of $-10,0$, and $+10 \mathrm{~cm} \mathrm{H}_{2} \mathrm{O}$.

studies with its fine and accurate closed-loop control of these parameters. ${ }^{8,29}$

While there exist several servo-controlled generators for forced oscillatory excitation of the respiratory system, most employ loud-speaker designs which limit the magnitude of the delivered flow to the subject and thus are not suitable for sustaining ventilation by themselves. ${ }^{3,5,7}$ As such, they must operate in parallel to existing flow or volume generators if they are to be used in ventilated patients. Those designs which are capable of delivering high amplitude flows must use powerful linear motors which not only can be mechanically inefficient and cumbersome to operate in clinical environments, but also require considerable electrical power. ${ }^{28}$ Thus, our intention for this device was to have a simple and efficient method to generate high fidelity flow and pressure waveforms in humans and large animals under conditions of sustained mean airway pressure. While the most apparent application for such a device would be as a research tool, particularly for the measurement of respiratory impedance, it may also be of use in clinical environments where HFV is desirable. In this paper, we have demonstrated that our device is capable of delivering broad-band, high amplitude oscillatory flows while maintaining a mechanical test load at a constant mean pressure. Our device has an additional advantage over traditional loud-speaker and linear motor systems in its ability to generate pressure oscillations under both positive and negative load pressures (Figs. 10 and 11). This may be useful in experiments involving negative pressure ventilation or control of pleural pressure. Moreover, the ability of the device to follow dynamic changes in desired mean airway pressure during sinusoidal oscillations (Fig. 11) makes it ideally suited for protocols involving the tracking effective airway caliber at different lung volumes. ${ }^{10}$ Finally, combining an exhalation valve system with our device as described in Ref. (14) would allow it to be used in a variety of different conventional ventilatory modalities, such as assist-control, SIMV, pressure control, or proportional assist ventilation. ${ }^{17}$

Proportional solenoid valves have been available for several years, ${ }^{17,31}$ but to our knowledge no other investigators have tested their oscillatory performance or incorporated them into a system such as ours to generate broad-band oscillatory flows. Current generation mechanical ventilators often use proportional solenoid valves to deliver standard clinical inspiratory flow waveforms (i.e., step, half-sine, decelerating ramp) to patients. However, we
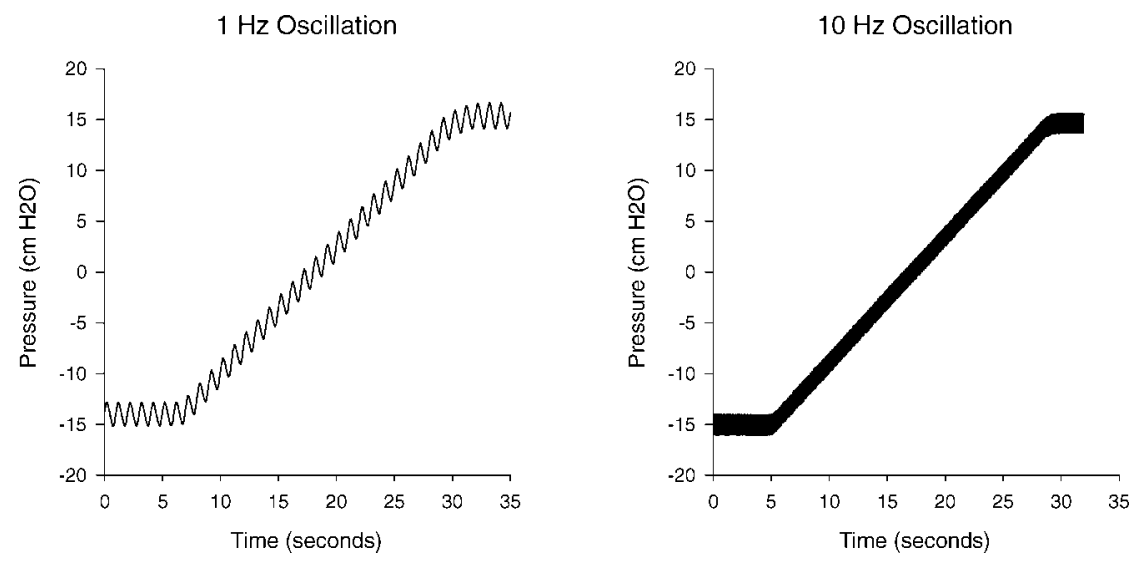

FIGURE 11. Actual pressure tracings for the resistor and glass bottle mechanical load at frequencies of 1.0 and $10.0 \mathrm{~Hz}$ while varying the desired mean pressure from -15 to $+15 \mathrm{~cm} \mathrm{H}_{2} \mathrm{O}$. 

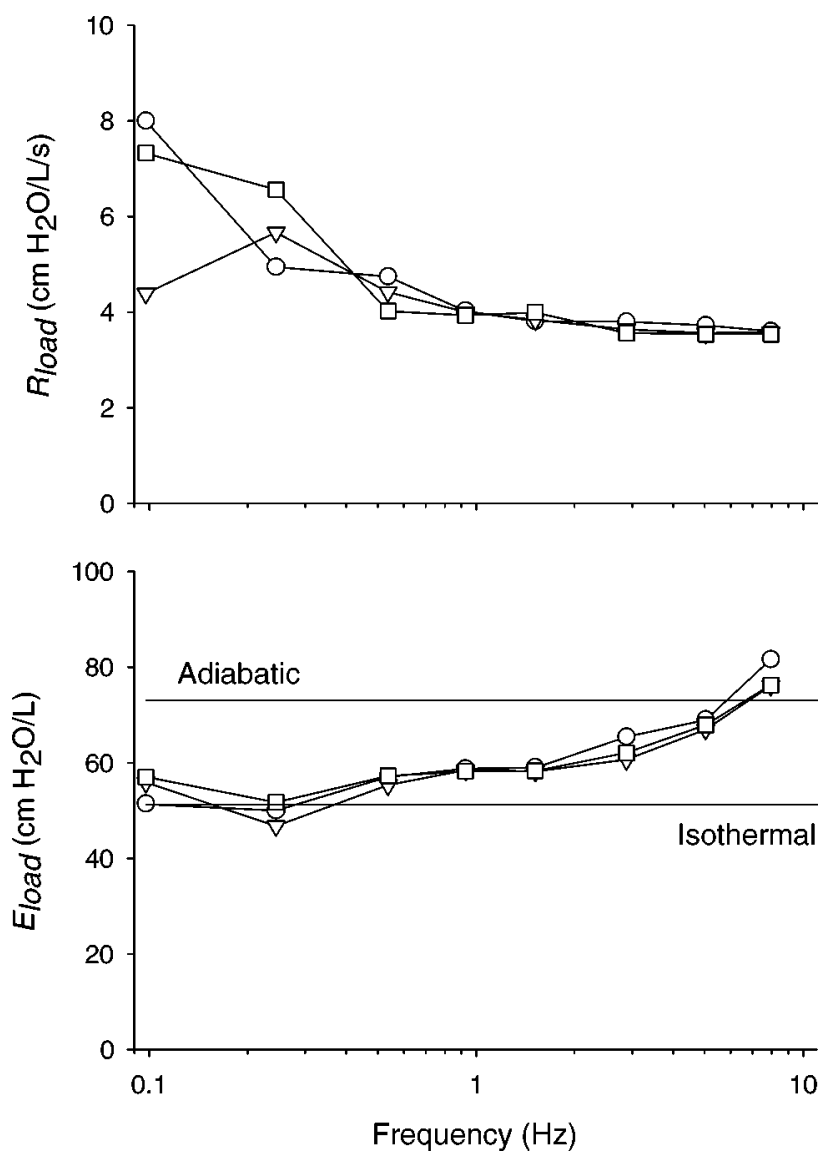

FIGURE 12. Mechanical test load resistance $\left(\boldsymbol{R}_{\text {load }}\right)$ and elastance ( $E_{\text {load }}$ ) versus frequency at mean load pressures of -10 (circles), 0 (inverted triangles), and +10 (squares) $\mathrm{cm} \mathrm{H}_{2} \mathrm{O}$. Solid lines represent the theoretical upper and lower limits of $E_{\text {load }}$ computed according to Eq. (2) assuming adiabatic compression at $+10 \mathrm{~cm} \mathrm{H}_{2} \mathrm{O}$ and isothermal compression at $-10 \mathrm{~cm} \mathrm{H} \mathrm{H}_{2} \mathrm{O}$.

are aware of no other pneumatically driven ventilator which is capable of delivering bidirectional broad-band oscillatory flows.

The steady-state voltage-flow relationship of the combined ECU-PSOL system does appear to be somewhat nonlinear, exhibiting both saturation nonlinearities as well as some mild hysteresis $(\sim 7 \%)$. This is most likely due to magnetic B-H nonlinearities of the solenoid and losses in its iron core. ${ }^{31}$ We cannot rule out that a portion of the nonlinearity near the maximum measured flow rate of $2.85 \mathrm{l} / \mathrm{s}$ may have arisen from the pneumotachograph, since the manufacturer guarantees $2 \%$ linearity only for bidirectional flows $<2.66 \mathrm{l} / \mathrm{s}$. However we subsequently learned that extending the maximum flow range by another $7 \%$ has minimal impact on the pneumotach's steady-state linearity (Hans Rudolph, Inc., personal communication).

In addition, the ECU-PSOL system demonstrated considerable dynamic nonlinearity in its output flow as assessed by the $k_{\mathrm{d}}$ index. This behavior may arise from distortions in the input driving signal, such as low signal-to-noise ratio, D/A quantization, and/or low-pass filter nonlinearities. While we did not make any attempt to correct for such input distortions, ${ }^{32}$ we feel that their impact on the output signal are minimal given the values of $k_{\mathrm{d}}$ for the input control voltage shown in Figs. 7 and 9. The larger values of $k_{\mathrm{d}}$ we observed in the output the flow under open-loop conditions may be secondary to nonlinearities intrinsic to the ECU-PSOL system and/or turbulent gas flow phenomena. The fact that the dynamic nonlinearity improved significantly when suction was applied to the output flow of the PSOL (Fig. 7) suggests that a portion of the nonlinear distortion may arise from turbulent effects, since suction reduces the output peak flow amplitude. While the harmonic distortion of the output flow may seem somewhat high (averaging 19.90 and $28.05 \%$ with and without a suction source, respectively), one must remember that the $k_{\mathrm{d}}$ index is dependent on bandwidth over which it is measured. ${ }^{32}$ In this study, our bandwidth was from 0.098 to $40.97 \mathrm{~Hz}$, considerably larger than the typical $0.1-10 \mathrm{~Hz}$ range used for employed for low frequency respiratory impedance measurements. ${ }^{12,13,15}$ Thus one may expect a reduction in harmonic distortion if such a smaller bandwidth is used.

The value of the experimentally determined $R_{\text {load }}$ for the test load demonstrated a trend of decreasing with increasing frequency, asymptotically approaching the value of the screen resistance of $4 \mathrm{~cm} \mathrm{H} \mathrm{H}_{2} \mathrm{O} / \mathrm{l} / \mathrm{s}$. Such behavior has been noted in previous studies using similar test loads, ${ }^{27}$ and is often attributed to a reduction in energy dissipation as gas compression transitions from an isothermal to adiabatic process as frequency is increased. ${ }^{25}$ Similarly, the energy storage associated with cyclic gas compression, as reflected in $E_{\text {load }}$, would be expected to increase with increasing frequency according to Eq. (2) as $\beta$ transitions from 1.0 to 1.4. However, since each of the applied frequencies in our broad-band NSND waveform was delivered to the load simultaneously, it is unlikely that gas compression can be characterized as a purely isothermal or adiabatic process at any one particular frequency.

Our device is distinct from traditional linear motor designs in that it incorporates the load impedance into the servo-loop. As such, the frequency response of the closedloop system will depend on the mechanical properties of the load impedance, which will vary from patient to patient. To assess the impact of different patient populations and pathophysiological conditions on the closed-loop performance of our device, we performed a Matlab computer simulation of the block diagram in Fig. 3. The details of this simulation are provided in the Appendix. The simulation was validated first by identifying an open-loop transfer function for the data of Fig. 6 and predicting the closedloop response for the entire system of Fig. 3 using a model $Z_{\text {load }}$ with parameters consistent with the screen resistor and bottle. Indeed, this prediction agreed remarkably well with the actual closed-loop frequency response as shown by the solid line in Fig. 8. Figure 13 shows the predicted 

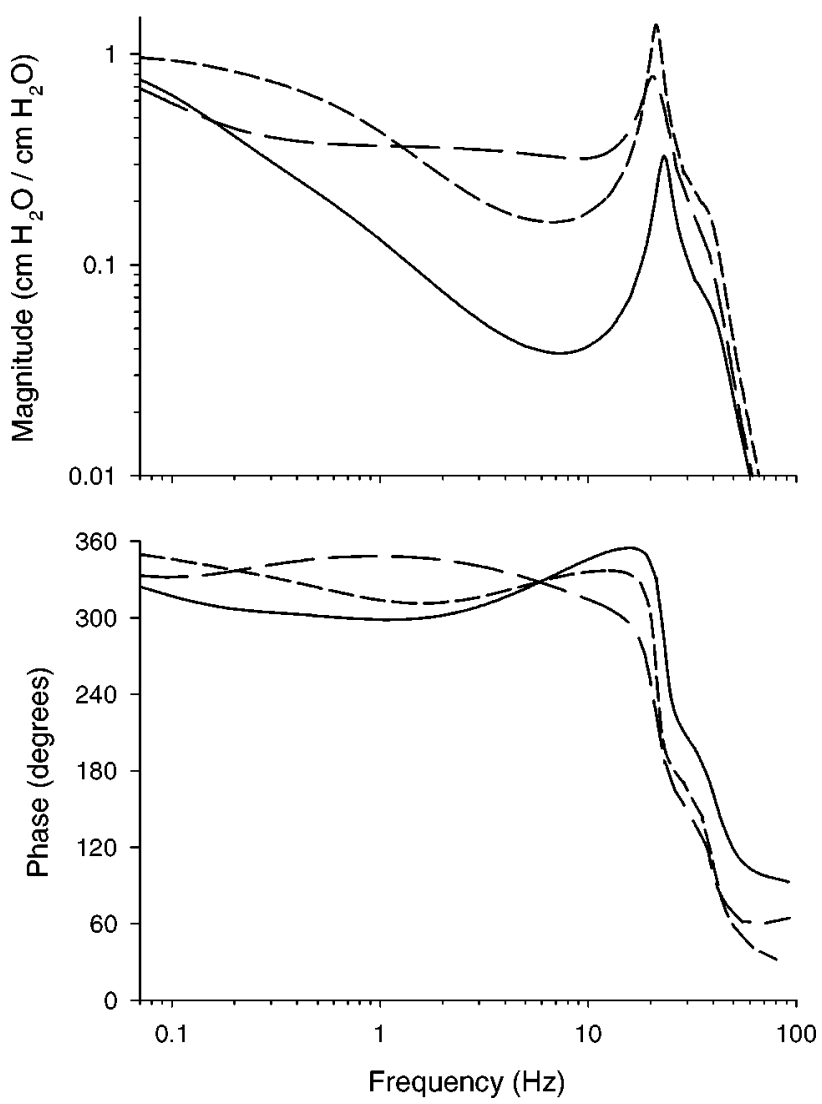

FIGURE 13. Simulated magnitude and phase response for the closed-loop pressure oscillator with load impedances adjusted to correspond to healthy adult (solid lines), healthy pediatric (short dashed line), and COPD (long dashed line) conditions. These simulations assume that load pressure is measured at distal end of the endotracheal tube.

magnitude and phase response of the closed-loop system with $Z_{\text {load }}$ adjusted to correspond to healthy adult, pediatric, and COPD conditions. While the magnitude demonstrated a significant roll-off from 0.1 to $10.0 \mathrm{~Hz}$ for both healthy adult and pediatric conditions, the predicted magnitude response for COPD patients was considerably flatter over this bandwidth, implying an improved frequency response of the system for these patients. Accordingly, we expect that the mechanical status of a patient's respiratory system will have significant influence on the dynamic behavior of this device. To compensate for this, one may adjust the analog proportional controller gain $K_{\mathrm{P}}$ and fine-tune the system performance from subject to subject. Alternatively, a PID controller could easily be incorporated into the device to further improve the system's frequency response. Since the pressure in the load is continuously sampled by an A/D board, a digital feedback controller could also be implemented and various discrete and/or adaptive controllers could be readily programmed to achieve any desired system dynamic requirements.

In summary, we have designed and implemented a device capable of delivering high amplitude, low frequency oscillatory flows while maintaining the load impedance at a specified mean pressure. In contrast to other devices capable of generating similar flows, ${ }^{15,28}$ our device does not rely on expensive linear motors driven by high-powered amplifiers. Thus it consumes minimal electrical power $(<23 \mathrm{~W})$ and occupies much less space compared to current devices capable of generating similar flows. The bandwidth of the device is sufficient for both low frequency respiratory mechanical impedance measurements as well as HFV. Moreover, this device could be used to measure the oscillatory flow response or impedance in many other systems, such as pipes, hollowed chambers, or other biological organs. Its ability to accurately control mean airway pressure, oscillation amplitude, and frequency content may make it ideal for use in clinical studies to optimize HFV protocols in patients. Future implementations of this device may incorporate PID or adaptive control for further improvements in its frequency response or response time.

\section{ACKNOWLEDGMENTS}

Supported by National Heart, Lung, and Blood Institute Grant R01 HL-62269-02 and HL-050515 and National Science Foundation Grant BES-9309426. The authors would like to thank Dr. Brett A. Simon for his many helpful suggestions during the preparation of this manuscript.

\section{APPENDIX}

In contrast to linear motor systems, our device (when operated under closed-loop conditions) controls pressure and not flow. In this situation, if one desires a specified flow waveform with adequate signal-to-noise ratio for the computation of respiratory impedance, the desired flow pattern must be converted to a corresponding pressure waveform as an input to the device. In principal, this requires a priori knowledge of the subject's respiratory impedance, although a rough prediction of delivered flow and/or tidal volume as well as the closed-loop frequency response of the oscillator can be obtained using representative impedance spectra from healthy or diseased lungs. ${ }^{12,13,15}$ This process can then be iterative or adaptive to maximize the fidelity of the delivered waveform. To illustrate this point, we simulated the closed-loop magnitude and phase response of the block diagram in Fig. 3 using the Matlab programming language (The MathWorks, Natick, MA). For these simulations, we experimentally determined the static gain of the Celesco LCVR0050 pressure transducer as $K_{\text {trans }}=0.0977 \mathrm{~V} / \mathrm{cm} \mathrm{H}_{2} \mathrm{O}$. An analog 8-pole Butterworth filter with a cut-off frequency of $50 \mathrm{~Hz}$ was simulated using the Matlab butter function. The gain of the proportional controller $K_{\mathrm{P}}$ was set to 0.3 as in our experimental protocol. We found that the frequency response of the open-loop ECU-PSOL system shown in Fig. 6 was adequately described by a 4 th order polynomial transfer 
TABLE A1. Parameter values for lumped element models used to simulated respiratory system impedance in healthy adult and pediatric patients as well as COPD patients.

\begin{tabular}{llccccc}
\hline Model topology & \multicolumn{1}{c}{ Condition } & $R_{1}$ & $I$ & $E_{1}$ & $R_{2}$ & $E_{2}$ \\
\hline Kelvin Body & Healthy adult & 1.79 & 0.011 & 31.21 & 5.35 & 17.52 \\
Kelvin Body & Healthy pediatric & 7.23 & 0.003 & 122.20 & 24.94 & 57.58 \\
Airway shunt & COPD adult & 4.40 & 0.010 & 723 & 28.40 & 31.81 \\
\hline
\end{tabular}

Note. Units: $R_{1}, R_{2}: \mathrm{cm} \mathrm{H} \mathrm{H}_{2} \mathrm{O} / \mathrm{l} / \mathrm{s} ; E_{1}, E_{2}: \mathrm{cm} \mathrm{H}_{2} \mathrm{O} / \mathrm{l} ;$ I: $\mathrm{cm} \mathrm{H}_{2} \mathrm{O} / / / \mathrm{s}^{2}$.

function, $T_{\mathrm{EP}}$ :

$$
T_{\mathrm{EP}}(s)=\frac{B_{0}}{s^{4}+A_{3} s^{3}+A_{2} s^{2}+A_{1} s+A_{0}}
$$

where $s$ is the complex Laplace domain variable. The coefficients of this transfer function were estimated from the data in Fig. 6 using a nonlinear gradient search technique, ${ }^{4}$ yielding the following values: $B_{0}=7.783 \times 10^{8}, A_{3}=$ $160.1, A_{2}=9.043 \times 10^{4}, A_{1}=4.832 \times 10^{6}, A_{0}=1.414$ $\times 10^{9}$. To predict what the closed-loop response of the device would be for the screen resistor and bottle load, we modeled $Z_{\text {load }}$ with a simple resistance and elastance series combination:

$$
Z_{\text {load }}(s)=R_{\text {load }}+\frac{E_{\text {load }}}{s}
$$

where $R_{\text {load }}=4.0 \mathrm{~cm} \mathrm{H}_{2} \mathrm{O} / \mathrm{l} / \mathrm{s}$ and $E_{\text {load }}=62.0 \mathrm{~cm} \mathrm{H}_{2} \mathrm{O} / 1$. To mimic polytropic gas compression, our value for $E_{\text {load }}$ was the average of its theoretical isothermal and adiabatic limits at atmospheric pressure as computed according to Eq. (2). While this model does not account for the frequency dependence of $R_{\text {load }}$ and $E_{\text {load }}$ seen in Fig. 12, we nonetheless found that its incorporation into the closed-loop simulation produced excellent agreement between the actual and predicted closed-loop frequency responses (Fig. 8).

To simulate the closed-loop response of the device in actual human subjects, we relied on two different lumped element models to describe $Z_{\text {load }}{ }^{14}$ For simulating healthy adult and pediatric respiratory impedance, we used a Kelvin-body model in which airway and Newtonian chest wall resistances are represented by element $R_{1}$, airway inertance by element $I$, and static linear tissue elastance by element $E_{1}$, all of which are in series with an $R_{2}-E_{2}$ parallel viscoelastic tissue combination. ${ }^{14}$ This model assumes that the frequency dependence of impedance is due solely to the viscoelastic properties of the lung parenchyma and chest wall. For patients with COPD, we relied on an airway shunt model originally proposed by $\mathrm{Mead}^{21}$ in which a central airway component consisting of $R_{1}$ and $I$ elements is separated from a peripheral airway resistance $R_{2}$ by a airway wall elastic element $E_{1}$. The combined elastances of the lungs and chest wall are represented by $E_{2}$. This model assumes that the dominant mechanism contributing to the frequency dependence of impedance is the shunting of flow into the central airway walls in the presence of increased peripheral airway resistance. Values for the elements of the Kelvin-body model (used for adult and pediatric simulations) and the airway shunt model (for COPD simulations) were derived from reported impedance data in the literature ${ }^{6,23,26}$ and are shown in Table A1. Both of these models admit the same form of polynomial transfer function:

$$
Z_{\text {load }}(s)=\frac{A s^{3}+B s^{2}+C s+D}{s(s+E)}
$$

the coefficients of which are algebraic functions of the model parameters and will depend on the model topology used. ${ }^{11,14}$ In order to avoid the influence of nonlinearities associated with endotracheal tubes, these simulations assume that the device is controlling tracheal pressure distal to the tube. ${ }^{24}$

\section{REFERENCES}

${ }^{1}$ Armstrong-Helouvry, B., P. DuPont, and C. C. de Wit. A survey of models, analysis tools and compensation methods for control of machines with friction. Automatica 30:1083-1138, 1994.

${ }^{2}$ Barnas, G. M., J. Sprung, T. M. Craft, J. E. Williams, I. G. Ryder, J. A. Yun, and C. F. Mackenzie. Effect of lung volume on lung resistance and elastance in awake subjects measured sinusoidal forcing. Anesthesiology 78:1082-1090, 1993.

${ }^{3}$ de Melo, P. L., M. M. Werneck, and A. Giannella-Neto. Linear servo-controlled pressure generator for forced oscillation measurements. Med. Biol. Eng. Comput. 36:11-16, 1998.

${ }^{4}$ Dennis, J. E., D. M. Gay, and R. E. Welsch. An adaptive nonlinear least-squares algorithm. ACM Trans. Math. Softw. 7:348-368, 1981.

${ }^{5}$ Farre, R., M. Ferrer, M. Rotger, and D. Navajas. Servocontrolled generator to measure respiratory impedance from 0.25 to $26 \mathrm{~Hz}$ in ventilated patients at different PEEP levels. Eur. Respir. J. 8:1222-1227, 1995.

${ }^{6}$ Farre, R., M. Ferrer, M. Rotger, A. Torres, and D. Navajas. Respiratory mechanics in ventilated COPD patients: Forced oscillation versus occlusion techniques. Eur. Respir. J. 12:170-176, 1998.

${ }^{7}$ Farre, R., M. Rotger, J. M. Montserrat, and D. Navajas. A system to generate simultaneous forced oscillation and continuous positive airway pressure. Eur. Respir. J. 10:1349-1353, 1997.

${ }^{8}$ Goddon, S., Y. Fujino, J. M. Hromi, and R. M. Kacmarek. Optimal mean airway pressure during high-frequency oscillation predicted by the pressure-volume curve. Anesthesiology $94: 862$ 869, 2001.

${ }^{9}$ Hantos, Z., B. Daroczy, B. Suki, G. Galgoczy, and T. Csendes. Forced oscillatory impedance of the respiratory system at low frequencies. J. Appl. Physiol. 60:123-132, 1986.

${ }^{10}$ Jensen, A., H. Atileh, B. Suki, E. P. Ingenito, and K. R. Lutchen. Selected Contribution: Airway caliber in healthy and asthmatic 
subjects: Effects of bronchial challenge and deep inspirations. $J$. Appl. Physiol. 91:506-515, 2001.

${ }^{11}$ Kaczka, D. W., G. M. Barnas, B. Suki, and K. R. Lutchen. Assessment of time-domain analyses for estimation of lowfrequency respiratory mechanical properties and impedance spectra. Ann. Biomed. Eng. 23:135-151, 1995.

${ }^{12}$ Kaczka, D. W., E. P. Ingenito, S. C. Body, S. E. Duffy, S. J. Mentzer, M. M. DeCamp, and K. R. Lutchen. Inspiratory lung impedance in COPD: Effects of PEEP and immediate impact of lung volume reduction surgery. J. Appl. Physiol. 90:1833-1841, 2001.

${ }^{13}$ Kaczka, D. W., E. P. Ingenito, E. Israel, and K. R. Lutchen. Airway and lung tissue mechanics in asthma: Effects of albuterol. Am. J. Respir. Crit. Care Med. 159:169-178, 1999.

${ }^{14}$ Kaczka, D. W., E. P. Ingenito, and K. R. Lutchen. Technique to determine inspiratory impedance during mechanical ventilation: Implications for flow-limited patients. Ann. Biomed. Eng. 27:340-355, 1999.

${ }^{15}$ Kaczka, D. W., E. P. Ingenito, B. Suki, and K. R. Lutchen. Partitioning airway and lung tissue resistances in humans: Effects of bronchoconstriction. J. Appl. Physiol. 82:1531-1541, 1997.

${ }^{16}$ Krishman, J. A., and R. G. Brower. High-frequency ventilation for acute lung injury and ARDS. Chest 118:795-807, 2000.

${ }^{17}$ Lua, A. C., K. C. Shi, and L. P. Chua. Proportional assist ventilation system based on proportional solenoid valve control. Med. Eng. Phys. 23:381-389, 2001.

${ }^{18}$ Lutchen, K. R., and H. Gillis. Relationship between heterogeneous changes in airway morphometry and lung resistance and elastance. J. Appl. Physiol. 83:1192-1201, 1997.

${ }^{19}$ Lutchen, K. R., Z. Hantos, and A. C. Jackson. Importance of low-frequency impedance data for reliably quantifying parallel inhomogeneities of respiratory mechanics. IEEE Trans. Biomed. Eng. 35:472-481, 1988.

${ }^{20}$ Lutchen, K. R., K. Yang, D. W. Kaczka, and B. Suki. Optimal ventilation waveforms for estimating low-frequency respiratory impedance. J. Appl. Physiol. 75:478-488, 1993.
${ }^{21}$ Mead, J. Contribution of compliance of airways to frequencydependent behavior of lungs. J. Appl. Physiol. 26:670-673, 1969.

${ }^{22}$ Michaelson, E. D., E. D. Grassman, and W. Peters. Pulmonary mechanics by spectral analysis of forced random noise. J. Clin. Invest. 56:1210-1230, 1975.

${ }^{23}$ Navajas, D., R. Farre, J. Canet, M. Rotger, and J. Sanchis. Respiratory input impedance in anesthetized paralyzed patients. J. Appl. Physiol. 69:1372-1379, 1990.

${ }^{24}$ Navajas, D., R. Farre, M. Rotger, and J. Canet. Recording pressure at the distal end of the endotracheal tube to measure respiratory impedance. Eur. Respir. J. 2:178-184, 1988.

${ }^{25}$ Peslin, R., and J. J. Fredberg. Oscillation mechanics of the respiratory system. In Handbook of Physiology the Respiratory System. Mechanics of Breathing. Bethesda: American Physiological Society, 1986, Sec. 3, Vol. III, Pt. 1, Chapter 11, pp. 145-178.

${ }^{26}$ Petak, F., B. Babik, T. Asztalos, G. L. Hall, Z. I. Deak, P. D. Sly, and Z. Hantos. Airway and tissue mechanics in anesthetized paralyzed children. Pediatr. Pulmonol. 35:169-176, 2003.

${ }^{27}$ Schuessler, T. F., and J. H. T. Bates. A computer-controlled research ventilator for small animals: Design and evaluation. IEEE Trans. Biomed. Eng. 42:860-866, 1995.

${ }^{28}$ Simon, B. A., and W. Mitzner. Design and calibration of a high-frequency oscillatory ventilator. IEEE Trans. Biomed. Eng. 38:214-218, 1991.

${ }^{29}$ Simon, B. A., G. G. Weinman, and W. Mitzner. Mean airway pressure and alveolar pressure during high-frequency ventilation. J. Appl. Physiol. 57:1069-1078, 1984.

${ }^{30}$ Suki, B., and K. R. Lutchen. Pseudorandom signals to estimate apparent transfer and coherence functions of nonlinear systems: Applications to respiratory mechanics. IEEE Trans. Biomed. Eng. 39:1142-1151, 1992.

${ }^{31}$ Vaughan, N. D., and J. B. Gamble. The modeling and simulation of a proportional solenoid valve. J. Dyn. Syst. Meas. Control 118:120-125, 1996.

${ }^{32}$ Zhang, Q., B. Suki, and K. R. Lutchen. Harmonic distortion from nonlinear systems with broadband inputs: Applications to lung mechanics. Ann. Biomed. Eng. 23:672-681, 1995. 